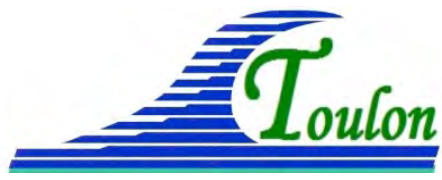
XIV $V^{e ̀ m e s}$ Journées Nationales Génie Côtier - Génie Civil Toulon, 29 juin au $1^{\text {er }}$ juillet 2016

DOI:10.5150/jngcgc.2016.064 (C) Editions Paralia CFL disponible en ligne - http://www.paralia.fr - available online

\title{
Analyse comparée des méthodes de surveillance de l’herbier de posidonies : cahier technique du gestionnaire
}

\section{Claire NOEL ${ }^{1}$, Pierre BOISSERY ${ }^{2}$, Valérie RAIMONDINO ${ }^{3}$, Nathalie QUELIN $^{4}$}

1. CARTOCEAN. Centre Associatif de Recherche et Technologie de l'OCEAN, 39 chemin de la Buge, 83110 Sanary s/Mer, France. noel@cartocean.fr

2. AERMC. Agence de l'Eau Rhône-Méditerranée et Corse. 62 La Canebière., 13001 Marseille, France. pierre.boissery@eaurmc.fr

3. Région PACA. Service Mer et Littoral. Région Provence-Alpes-Côte-d'Azur., 27, place Jules-Guesde, Marseille cedex 20, 13481, France. vraimondino@regionpaca.fr

4. DREAL PACA. Direction Régionale de l'Environnement, de l'Aménagement et du Logement PACA. Site du Tholonet. CS80065, 13182 Aix en Provence Cedex 5, France.

nathalie.quelin@developpement-durable.gouv.fr

\section{Résumé :}

Ce cahier rappelle le besoin de connaissances nécessaires en termes de surveillance des herbiers de posidonies. Il présente les outils et les méthodes disponibles pour mettre en place une surveillance (ou un suivi) des herbiers adaptée aux champs de compétences des gestionnaires et aux problématiques de leur territoire.

Les résultats de nos travaux d'analyse comparée des méthodes de surveillance de l'herbier de posidonies sont présentés dans un cahier technique, document de 96 pages, pédagogique et illustré qui s'adresse aux élus, gestionnaires et techniciens qui gèrent un espace maritime public ou privé, et/ou souhaitent l'aménager.

Mots-clés : Monitoring des herbiers de posidonies, Génie côtier, GIZC, Etude d'impact environnementaux, Sédiments, Travaux maritimes, Environnement littoral, Ecosystèmes côtiers.

\section{Introduction}

Si cartographier le fond marin était jusqu’à présent une finalité, les directives européennes demandent maintenant que cette finalité soit orientée vers le contrôle de l'obtention du bon état écologique. Il devient alors nécessaire de disposer de séries cartographiques itératives par zone, et d'en analyser l'évolution, c'est-à dire d'effectuer un « monitoring » ou une surveillance des habitats marins.

Le travail de monitoring est beaucoup plus complexe que le travail de cartographie. Il nécessite une approche rigoureuse en termes de respect de la chaine de " précision- 


\section{Thème 6 - Gestion durable des zones littorales et estuariennes}

résolution » des données cartographiées. Si cette chaine n'est pas respectée, ou si la méthode utilisée n'est pas adaptée à la problématique, l'évolution de l'état écologique des habitats sera mal mesurée et chiffrée, et les conclusions en termes de mesures de gestion mises en œuvre peuvent s'avérer au pire désastreuses, au mieux inutiles.

Ce guide propose une méthodologie d'analyse rigoureuse et chiffrée des technologies de monitoring existantes.

La démarche préconisée correspond à celle actuellement mise en œuvre dans le monde médical, et ce depuis que de nouvelles technologies performantes d'analyses surfaciques et volumiques du corps humain sont disponibles.

Pourquoi un cahier technique sur le suivi ? Tout projet d'aménagement est instruit par les services de l'État, la Direction Départementale des Territoires et de la Mer (DDTM) qui demandent une analyse préalable de l'impact sur les herbiers, et de l'environnement plus généralement, voire un suivi spécifique de l'herbier.

Ce cahier présente un cadre de référence. Il préconise les moyens à mettre en œuvre en termes de suivi. Ces moyens doivent être économiquement faisables et les plus simples possibles, tout en satisfaisant aux objectifs de connaissances.

Ces dernières années, les technologies ont beaucoup évolué et ont vu en particulier l'avènement de nouvelles méthodes de cartographie et de suivi (ou de monitoring) qui permettent une approche à la fois plus large et plus précise, et à des coûts abordables, que l'on peut donc mettre en œuvre plus souvent et avec une grande précision, aboutissant à un suivi scientifique plus complet et plus performant de l'évolution des herbiers. Tout en restant simples, ces nouveaux outils permettent de compléter les technologies plus anciennes qui ont montré leurs limites, notamment en ce qui concerne la faible superficie de l'herbier qu'elles permettaient d'étudier et la difficulté de synthétiser un ensemble de données ponctuelles.

En parallèle de ces méthodes, de nouveaux outils, permettant une consultation aisée des données et une bancarisation simplifiée, ont été mis au point. Ces outils exploitent des formats standardisés et pérennes. Ils sont conviviaux et facilement utilisables par tout public, expert ou non. Ils permettent à faible coût (voire gratuitement) de diffuser et de consulter les données sans SIG, ni qualification spécifique en sciences de la terre.

Ce cahier technique (NOËL et al., 2013) :

a) rappelle les besoins de suivi de l'herbier de posidonies: son rôle écologique, les pressions qu'il subit et leur impact, les réglementations qui le protègent (Chapitre 1) ;

b) explicite les notions scientifiques nécessaires à la compréhension des méthodes de suivi (Chapitre 2);

c) présente la boite à outils de suivi disponible, c'est-à-dire les technologies de suivi (Chapitre 3);

d) préconise enfin, et surtout, les méthodes les plus adaptées à chaque typologie d'impact (Chapitre 4). 


\section{XIV èmes Journées Nationales Génie Côtier - Génie Civil \\ Toulon, 29 juin au $1^{\text {er }}$ juillet 2016}

\section{Matériel et méthodes}

Ce cahier se veut être un outil d'aide à la gestion, pragmatique et à la portée des élus, gestionnaires et techniciens. Il fait le point sur les méthodes existantes et vise à compléter le guide "Préservation et conservation des herbiers à Posidonia oceanica" (BOUDOURESQUE et al.. 2006) réalisé dans le cadre de l'Accord RAMOGE entre la France, l'Italie et Monaco.

Nos travaux d'analyse comparée des méthodes de surveillance de l'herbier de posidonies se sont basés :

a) une importante bibliographie sur le sujet (NOËL et al., 2013)

b) des entretiens auprès des spécialistes du domaine : institutions, gestionnaires locaux, bureaux d'études et scientifiques.

c) une approche cartésienne et chiffrée : En raison de la grande différence de nature des méthodes de suivi ponctuelles, linéaires, micro-surfaciques et surfaciques, nous ne pouvons procéder à leur comparaison qu'en définissant des critères communs, des niveaux de performances et des valeurs seuils correspondantes, même si les unités ne sont pas les mêmes. Dans cette phase nous avons explicité les paramètres des méthodes de surveillance, avant de définir les critères qui caractérisent chaque méthode et permettent d'en analyser les performances.

\section{Résultats}

Nous présentons une synthèse des différentes méthodes d'observation de la répartition spatiale et de la vitalité de l'herbier de posidonies disponibles, ainsi que leurs performances.

L'analyse comparée des méthodes de surveillance de l'herbier de posidonies aboutit à la préconisation d'une démarche de surveillance qui repose :

a) sur une approche globale surfacique de la couverture de l'herbier, accessible en raison de l'avènement récent de nouvelles technologies,

b) suivie d'une approche surfacique locale ou d'une approche ponctuelle (en station) ciblée de sa vitalité, dès lors que les impacts sont bien localisés.

Les figures 1 et 2 présentent pour chaque méthode, les suivis d'impact pour lesquels elle est particulièrement performante.

L'adéquation de leurs performances en regard des objectifs de la surveillance, permet dans le dernier chapitre de proposer pour chaque type d'impact les méthodes les mieux adaptées. 
Thème 6 - Gestion durable des zones littorales et estuariennes

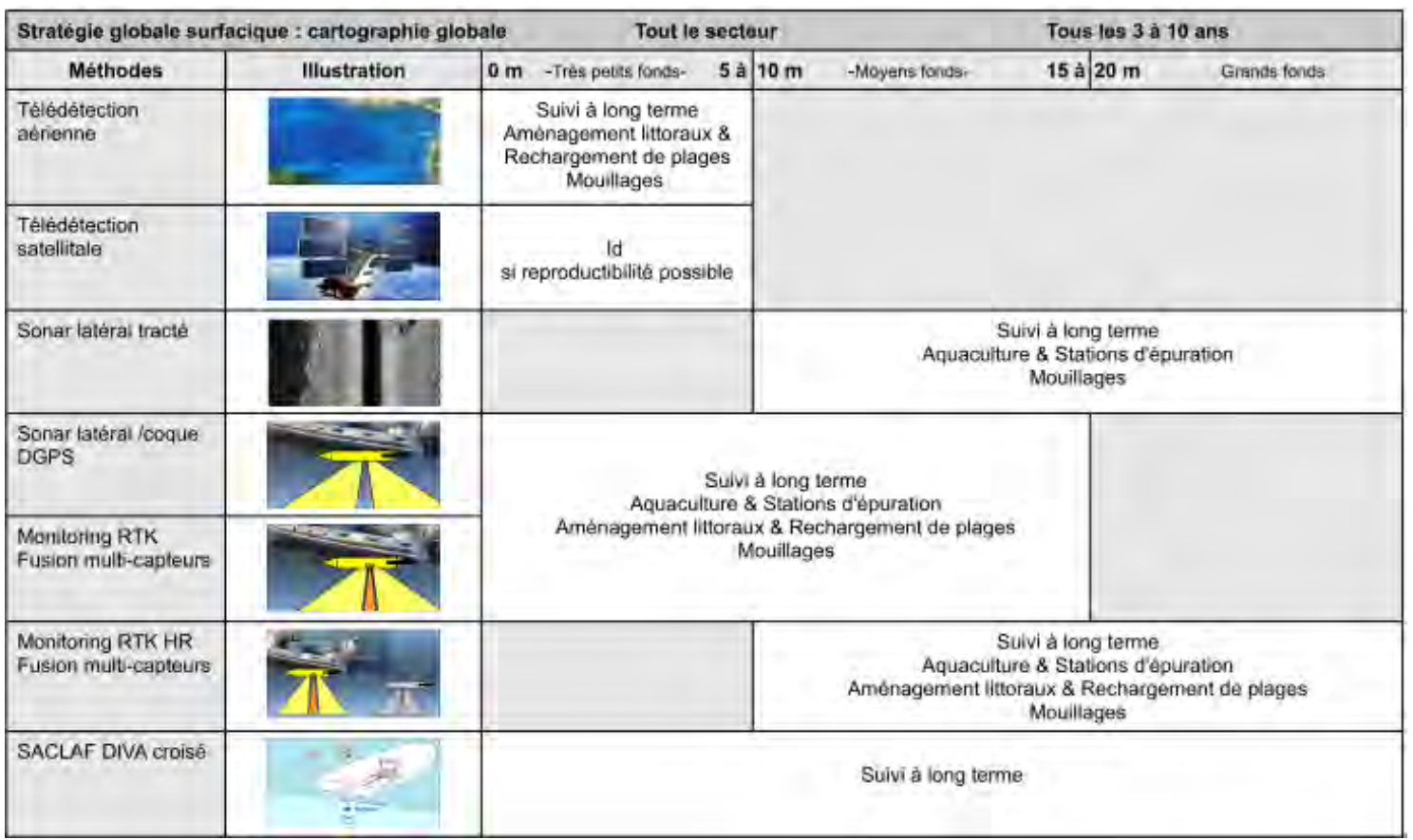

Figure 1. Méthodes globales de monitoring et suivis d'impact pour lesquelles elles sont particulièrement performantes.

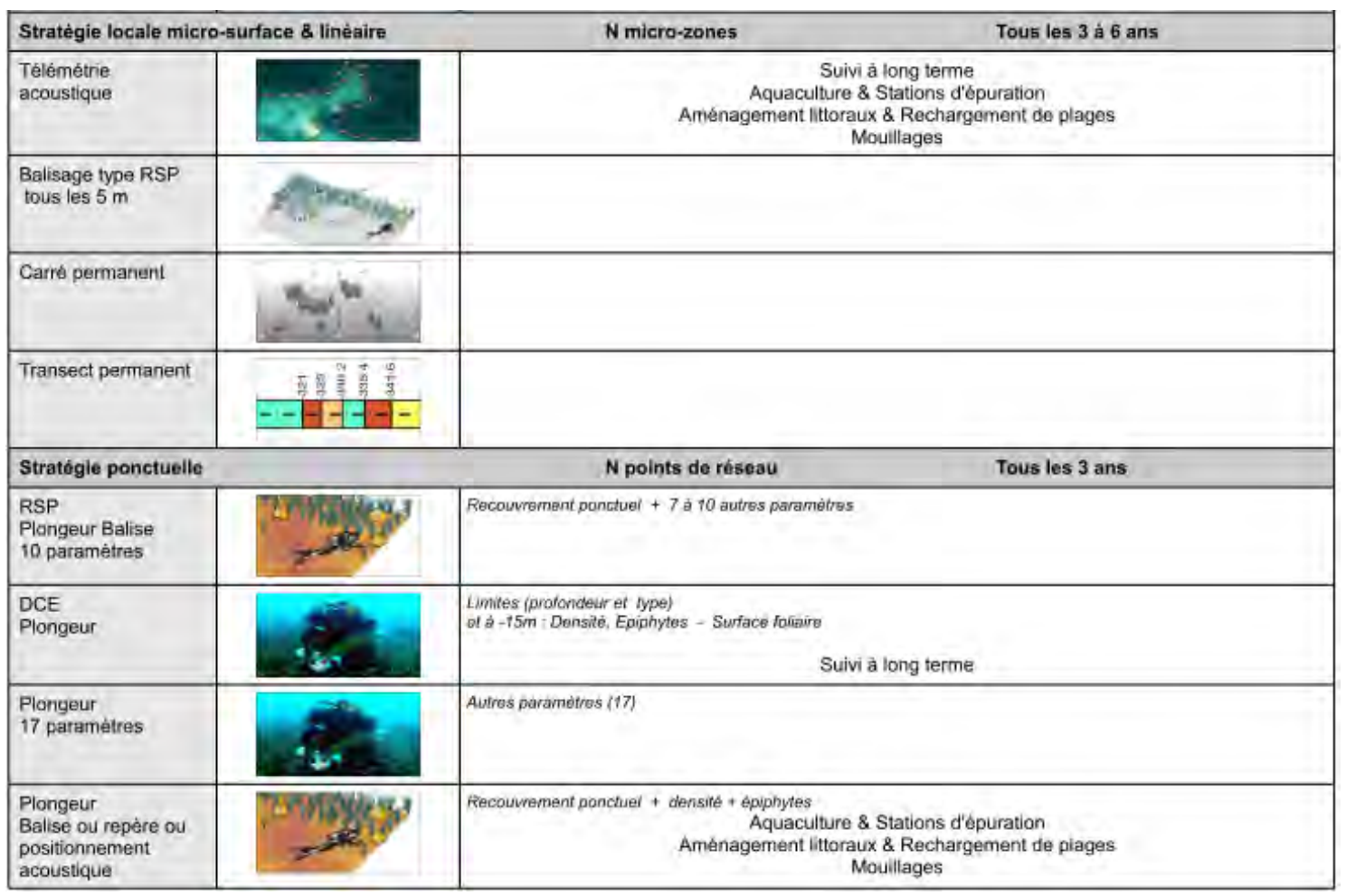

Figure 2. Méthodes de monitoring locales et stationnelles et suivis d'impact pour lesquelles elles sont particulièrement performantes. 


\section{XIV èmes Journées Nationales Génie Côtier - Génie Civil \\ Toulon, 29 juin au $1^{\text {er }}$ juillet 2016}

\section{Conclusions}

La démarche préconisée (voir figure 3) correspond à celle actuellement mise en œuvre dans le monde médical, et ce depuis que de nouvelles technologies performantes d'analyses surfaciques et volumiques du corps humain sont disponibles. Tout comme une prise de sang ou une biopsie ne permettent pas de surveiller la santé de l'homme, une analyse stationnelle ne peut pas, à elle seule, permettre de surveiller la santé de l'herbier ; ces analyses ponctuelles sont effectuées dans un second temps, lorsque l'approche globale (imagerie médicale) en a démontré la nécessité et après qu'elle ait mis en évidence les zones à surveiller.

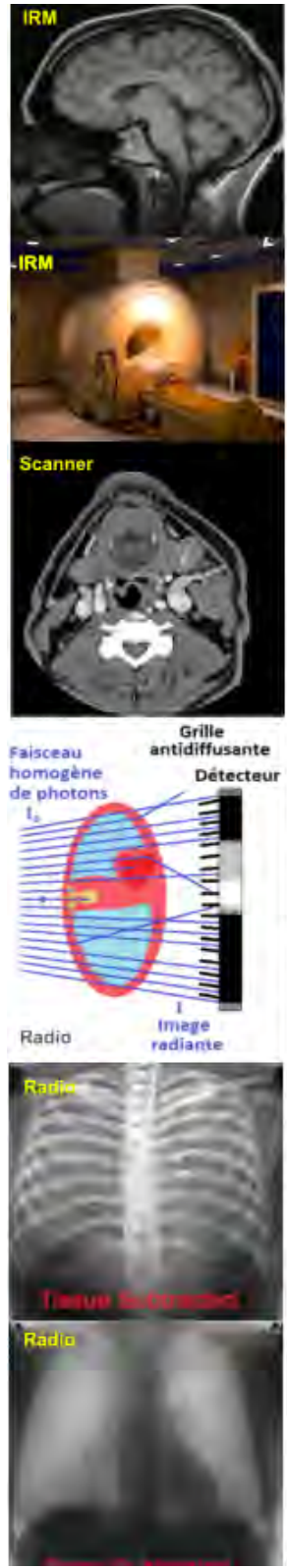

\begin{tabular}{|c|c|}
\hline Surveillance de la santé de l'homme & Suivi de la santé de l'herbier \\
\hline \multicolumn{2}{|c|}{ A une date $\mathrm{T}$} \\
\hline $\begin{array}{l}\text { Le médecin commande une analyse } \\
\text { surfacique auprés du spécialiste, qui est } \\
\text { un radiologue. } \\
\text { Le radiologue maitrise le fonctionnement de } \\
\text { ses appareillages et réalise les acquisitions. } \\
\text { - scanner } \\
\text { - radiométrie } \\
\text { - IRM } \\
\text {-... }\end{array}$ & 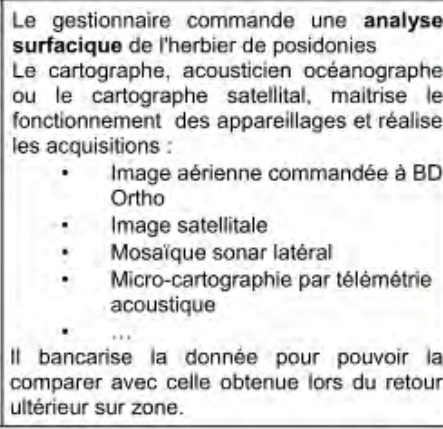 \\
\hline \multicolumn{2}{|c|}{ Pré-analyse des images } \\
\hline $\begin{array}{c}\text { Repérage des zones non-homogènes } \\
\text { suspectes : } \\
\begin{array}{l}\text { Définition de zones à contrôler } \\
\text { Spécifications des examens } \\
\text { complémentaires invasifs }\end{array} \\
\end{array}$ & \begin{tabular}{|l} 
Segmentation des zones homogènes : \\
Choix de la stratégie \\
d'èchantillonnage \\
Définition du plan \\
d'échantillonnage
\end{tabular} \\
\hline $\begin{array}{l}\text { Réalisation des prélèvements effectués par } \\
\text { le biologiste : } \\
\begin{array}{l}\text { prise de sang } \\
\text { biopsie... }\end{array}\end{array}$ & $\begin{array}{l}\text { Réalisation des vérités terrain effectuèes } \\
\text { par le biologiste } \\
\begin{array}{c}\text {. } \\
\text { observations } \\
\text { mesures de la vitalité ... }\end{array}\end{array}$ \\
\hline \multicolumn{2}{|c|}{ Interprétation des images } \\
\hline $\begin{array}{l}\text { effectuée par le radiologue } \\
\text { - Identification et classification des } \\
\text { zone suspectes } \\
\text { Mesure de la surface et de } \\
\text { l'étendue de ces zones } \\
\text { (tumeurs...) }\end{array}$ & $\begin{array}{l}\text { effectuée par le cartographe } \\
\text { - } \quad \text { classification des zones } \\
\text { homogènes } \\
\text { - } \quad \text { production de la cartographie } \\
\text { - mesure des étendues }\end{array}$ \\
\hline \multicolumn{2}{|c|}{$\begin{array}{l}\text { Définition du protocole de suivi : } \\
\text { zone à surveiller et récurrence temporelle }\end{array}$} \\
\hline \multicolumn{2}{|c|}{ A la date $T+$ récurrence } \\
\hline $\begin{array}{l}\text { Nouveaux examens } \\
\text { - } \quad \text { imagerie } \\
\text { - prélèvements }\end{array}$ & $\begin{array}{l}\text { Retour sur zone } \\
\text { - imagerie } \\
\text { observations, mesures de la } \\
\text { vitalité }\end{array}$ \\
\hline 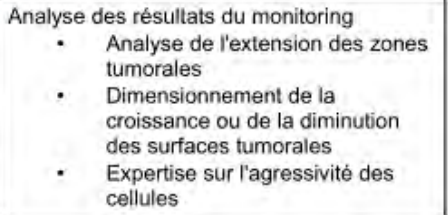 & $\begin{array}{l}\text { Analyse des résultats du monitoring } \\
\text { Analyse de l'extension ou de la } \\
\text { régression des surfaces d'herbier } \\
\text { Chiffrage gråce à l'indice de } \\
\text { conservation } \\
\text { Expertise sur l'évolution des } \\
\text { paramétres ponctuels de la santé } \\
\text { de l'herbier }\end{array}$ \\
\hline
\end{tabular}

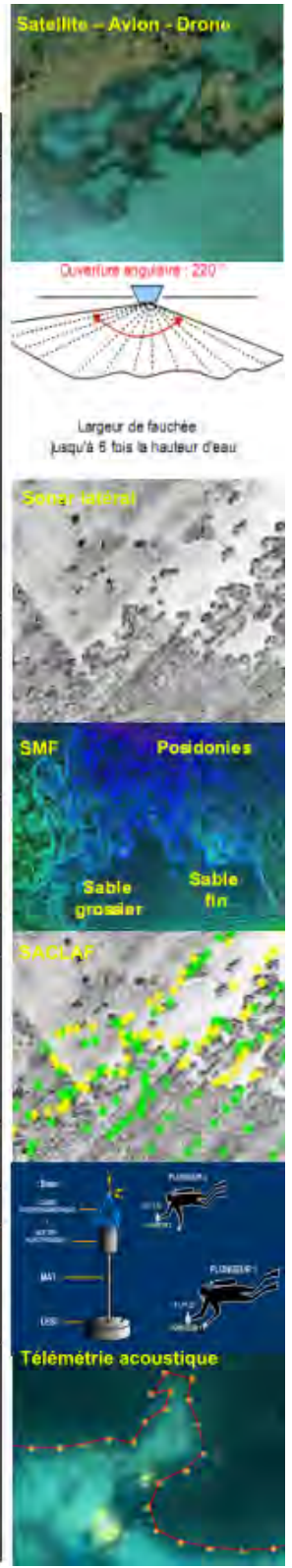

Figure 3. Démarche de suivi préconisée par le cahier technique. 
Thème 6 - Gestion durable des zones littorales et estuariennes

Il est important de noter, que bien que ces travaux aient été réalisés dans le cadre de suivi de l'herbier de posidonies, les résultats obtenus et la méthodologie utilisée dans l'analyse comparée s'appliquent de façon plus générale au suivi d'un fond marin ou aquatique.

\section{Références bibliographiques}

BOUDOURESQUE C.F., BERNARD G., BONHOMME P., CHARBONNEL E., DIVIACCO G., MEINESZ A., PERGENT G., PERGENT -MARTINI C., RUITTON S., TUNESI L., (2006.) Préservation et conservation des herbiers à Posidonia oceanica. Ramoge pub., Monaco: $202 \mathrm{p}$.

NOEL C., BOISSERY P., RAIMONDINO V., QUELIN N. (2013). Analyse comparée des méthodes de surveillance de l'herbier de posidonies: Cahier technique du gestionnaire. Edition CartOcean. 96 p. Disponible en ligne sur : http://cartocean.fr/pages/Cahier_Technique_Methodes_Suivi.htm - La bibliographie utilisée pour réaliser le cahier technique est elle aussi consultable en ligne : http://cartocean.fr/pages/biblio.htm 